\title{
Diversité, priorité pastorale et de conservation des ligneux fourragers des terres de parcours en zone soudano- guinéenne du Bénin
}

\author{
Clément Sèwadé ${ }^{(1)}$, Akomian Fortuné Azihou ${ }^{(1)}$, Adandé Belarmain Fandohan ${ }^{(1,2,3)}$, \\ Thierry Dèhouégnon Houéhanou ${ }^{(1,4)}$, Marcel Houinato ${ }^{(1)}$ \\ (1) Université d'Abomey-Calavi (UAC). Faculté des Sciences Agronomiques (FSA). Laboratoire d'Écologie Appliquée \\ (LEA).01 BP 526 Cotonou (Bénin).E-mail : sewadec@yahoo.fr \\ (2) Université d'Agriculture de Kétou. Unité de Foresterie, Agroforesterie et Biogéographie. École de Foresterie et Ingénierie \\ du Bois. BP 95. Kétou (Bénin). \\ ${ }^{(3)}$ Albert-Ludwigs-Universität Freiburg. Abteilung für Biometrie und Umweltsystemanalyse. Tennenbacher Str. 4. DE- \\ Freiburg 79085 (Deutschland). \\ (4) Université de Parakou. École Nationale Supérieure des Sciences et Techniques Agronomiques de Djougou. BP 73. \\ Djougou (Bénin).
}

Reçu le 19 juin 2015, accepté le 17 décembre 2015.

\begin{abstract}
Description du sujet. Les ligneux fourragers constituent une source importante pour la survie des ruminants en période de soudure. Vu la pression qu'ils subissent et par conséquent leur rareté dans les terres de parcours, une étude a été conduite dans la zone de transition soudano-guinéenne du Bénin auprès des populations riveraines des forêts classées des Monts Kouffé, de Wari-Maro et de l'Ouémé supérieur.

Objectifs. L'étude vise à identifier les ligneux fourragers prioritaires pour le pastoralisme, analyser l'utilisation et les intensités de coupe des ligneux fourragers en relation avec les différents groupes sociolinguistiques de la zone d'étude et évaluer leur priorité pour la conservation.
\end{abstract}

Méthode. Les données de la littérature ont permis de compléter celles issues des enquêtes afin de constituer une base de données sur différents critères définis. Les taux de citation des espèces par les populations ont permis d'établir leur priorité pour le pastoralisme, alors que la priorisation des ligneux pour la conservation est réalisée avec une combinaison de quatre méthodes et neuf critères.

Résultats. Les résultats montrent que 48 espèces sont utilisées comme fourrage aérien et réparties en 17 familles dominées par les Leguminosae $(27,1 \%)$ et les Moraceae (16,6\%), et 37 genres dont le plus représenté est le genre Ficus $(16,6 \%)$. L'appétibilité, la disponibilité des espèces et leur productivité (au niveau des animaux) sont les critères de choix des ligneux fourragers par les différents groupes sociolinguistiques. Afzelia africana, Pterocarpus erinaceus, Khaya senegalensis, Vitellaria paradoxa, Mangifera indica, Ficus platyphylla, Balanites aegyptiaca, Annona senegalensis, Ficus umbellata et Daniellia oliveri sont les dix premiers ligneux fourragers prioritaires identifiés pour la conservation dont la plupart se trouvent prioritaires pour le pastoralisme.

Conclusions. Pour une exploitation rationnelle des ligneux fourragers, nous suggérons l'utilisation des espèces prioritaires aussi bien pour le pastoralisme que pour la conservation dans les plans d'aménagement des parcours naturels au cours des activités d'afforestation/reforestation et de reboisement.

Mots-clés. Conservation de la diversité biologique, plante d'abroutissement, pastoralisme, ruminant, enquête, critère de sélection, Bénin.

\section{Diversity, pastoral and conservation priorities of fodder trees in the Sudano-Guinean pasture lands of Benin}

Description of the subject. Fodder trees are important for livestock survival in dryland Africa. In view of the pressure faced by these trees, and their consequent rarity noted in rangelands, a study was conducted in the Sudano-Guinean transition zone of Benin at the level of the local population surrounding the protected forests of Monts Kouffé, Wari-Maro and Ouémé Supérieur. Objectives. This study aimed to inventory the fodder trees, analyze the local perception of factors threatening target fodder trees, according to different sociolinguistic groups and prioritize fodder trees for conservation. 
Method. Ethnobiological surveys and ecological data from the available literature were used to construct a database following different criteria. The citation rates of the fodder trees by the surveyed populations were used to establish pastoral priority, while their conservation priority was established using a combination of four methods and nine criteria.

Results. Forty-eight fodder trees belonging to 17 families dominated by Leguminosae (27.1\%) and Moraceae (16.6\%) were reported. These species were distributed among 37 genera, with the genus Ficus being the most represented (16.6\%). Palatability, species availability and the impact of tree fodder on animal productivity were the criteria used by the surveyed sociolinguistic groups in their selection of fodder trees. The prioritization methods yielded ten top ranked species: Afzelia africana, Pterocarpus erinaceus, Khaya senegalensis, Vitellaria paradoxa, Mangifera indica, Ficus platyphylla, Balanites aegyptiaca, Annona senegalensis, Ficus umbellata and Daniellia oliveri.

Conclusions. With the aim of establishing the sustainable management of pasture lands, we suggest that priority be given to the aforementioned species of fodder trees as part of restoration, afforestation/reforestation and plantation activities.

Keywords. Biodiversity conservation, browse plants, pastoralism, ruminants, surveys, selection criteria, Benin.

\section{INTRODUCTION}

L'élevage animal en général et celui des ruminants en particulier joue un rôle central dans l'économie des pays ouest-africains avec une contribution au PIB agricole allant de $5 \%$ à $44 \%$ pour les pays du Sahel (Kamuanga et al., 2008 ; FAO, 2009). Sa participation au PIB du Bénin était estimée à 6,2 \% (MDR, 1994).

Les arbres et arbustes fourragers, utilisés comme compléments de la ration des ruminants, constituent des moyens de survie pour le bétail, surtout pendant la saison sèche. Ils participent à l'entretien aussi bien des animaux domestiques que des troupeaux transhumants dans les milieux arides et semi-arides dont la période sèche peut s'étendre sur sept mois (Sarr et al., 2013). L'utilisation des ligneux fourragers pour alimenter les animaux, surtout en saison sèche, a été signalée par plusieurs auteurs en Éthiopie (Abule, 2004), au Nigéria (Tukur et al., 2013), au Bénin (Houinato, 2001 ; Brisso et al., 2007 ; Houéhanou et al., 2008), au Burkina Faso (Kaboré-Zoungrana, 1995 ; Petit et al., 2000 ; Sanon et al., 2008 ; Soulama et al., 2013), au Sénégal (Sarr et al., 2013), au Cameroun (Onana et al., 2002) et au Tchad (Bechir et al., 2009). Les espèces les plus citées par ces auteurs sont Khaya senegalensis, Afzelia africana, Prosopis africana et Pterocarpus erinaceus. Cette utilisation assez largement répandue des ligneux fourragers est favorisée non seulement par la palatabilité de leurs feuilles mais aussi par le caractère sempervirent ou semi-sempervirent de ces espèces (Hiernaux et al., 1994 ; Baumer, 1997 ; Hiernaux, 2000).

Dans les zones soudanienne et soudano-guinéenne du Bénin où l'élevage des ruminants (surtout des bovins) est pratiqué, des difficultés d'alimentation des animaux se posent avec acuité, surtout en saison sèche, où il n'y a pas de fourrages herbacés, aussi bien naturels qu'artificiels qui, pour ces derniers, n'existent quasiment pas dans ces régions. Dès lors, l'alimentation des animaux élevés par les communautés locales et de ceux des transhumants, repose intégralement sur la cueillette des ligneux fourragers des aires de parcours, sans efforts d'aménagement et de renouvellement soutenu. Cela exerce une pression sur les ligneux qui se font de plus en plus rares. De plus, la végétation naturelle est de plus en plus menacée par d'autres facteurs tels que l'urbanisation, l'agriculture, la carbonisation et l'exploitation forestière.L'exploitation forestière, qui s'intéresse aux essences de valeur qui sont aussi souvent utilisées comme ligneux fourragers, ainsi que l'expansion de la culture cotonnière, réduisent davantage le potentiel de fourrage aérien disponible. Le rajeunissement et la diversification assistée de ces pâturage (ex. par la reforestation) pourraient contribuer à atténuer la pression actuelle sur ces ligneux fourragers sur les terres de parcours et renforcer la capacité de charge de ces derniers. La réussite d'une telle intervention est tributaire d'une bonne connaissance de la diversité originelle des pâturages et des préférences des animaux. Les préférences des ligneux fourragers par les animaux sur les terres de parcours sont conditionnées par plusieurs facteurs. La disponibilité des essences les plus appétées et les besoins de productivité amènent les éleveurs à sélectionner les espèces de fourrage ligneux. Par exemple, au Népal, les éleveurs utilisent prioritairement les essences qui améliorent, selon eux, la production laitière des animaux, notamment Bauhinia purpurea, Artocarpus lakoocha, Ficus lacor, Leucaena leucocephala, Ficus cunia (Barshila et al., 2013). Ahmad et al. (2009) ont procédé à une priorisation des ligneux en se basant sur leur valeur médicinale pour les populations riveraines du parc national de Margala Hills à Islamabad au Pakistan. Roothaert et al. (2001) ont noté que les préférences des ligneux fourragers par les éleveurs au Kenya varient suivant les zones agroécologiques. Ainsi, la capacité des ligneux à satisfaire le besoin alimentaire des animaux constitue le critère utilisé pour leur priorisation.

Pour une restauration écologique des milieux pouvant rendre disponibles les ligneux les plus utilisés, d'autres dimensions doivent être prises en compte comme, par exemple, la disponibilité de données sur 
la sylviculture des espèces concernées, leur statut de conservation, leur capacité de régénération ainsi que les différents facteurs qui influencent leur survie. Puisque cette restauration aura un cout, il serait bon de procéder à une priorisation des ligneux pour sélectionner ceux qui nécessitent plus d'attentions dans les stratégies de gestion rationnelle des ressources. Les ligneux fourragers, bien qu'étant recensés par plusieurs auteurs (Houéhanou et al., 2008), n'ont pas encore fait l'objet de priorisation, ni du point de vue pastoral, ni pour la conservation en Afrique de l'Ouest en général et au Bénin en particulier.

Différentes méthodes sont utilisées de par le monde pour hiérarchiser certains ligneux avec des critères variés. Certains travaux se sont intéressés à la priorisation des habitats et des communautés végétales en se basant sur leur richesse spécifique, l'origine, l'endémisme, l'importance économique et le statut de conservation des espèces qu'ils abritent (Joshi et al., 2004 ; Pant et al., 2007 ; Singh et al., 2010). Par contre, d'autres abordent directement la priorisation des espèces sur la base d'une combinaison de critères (Roothaert et al., 2001 ; Brehm et al., 2010 ; Idohou et al., 2013). Selon Brehm et al. (2010), il est préférable d'adopter une priorisation des espèces puisqu'elle permet aux conservateurs de connaitre les taxons à privilégier dans les efforts de conservation et ceux qui n'ont pas une importance immédiate avérée. La notion de conservation dans cet article ne s'identifie pas à une situation de restriction totale de l'utilisation des ressources par prélèvement mais plutôt à une utilisation rationnelle des ligneux fourragers assurant ainsi leur pérennité. L'objectif de cette étude est d'évaluer la diversité et la priorité pastorale et de conservation des ligneux fourragers des terres de parcours de la zone soudano-guinéenne du Bénin. Spécifiquement, il s'agit de :

- identifier les ligneux fourragers prioritaires ;

- analyser l'utilisation et les intensités de coupe des ligneux fourragers en relation avec les différents groupes sociolinguistiques de la zone d'étude ; - évaluer leur priorité pour la conservation.

Les hypothèses suivantes ont été formulées dans le cadre de cette étude. La première hypothèse stipule qu'en raison du manque d'eau et de fourrage en saison sèche, les éleveurs auraient davantage recours aux ligneux fourragers ayant des affinités pour les milieux humides (zone de bas-fond, forêt marécageuse, forêt galerie) et de ce fait, ces derniers subiraient plus de pression pastorale que d'autres. La seconde considère que les ligneux de la famille des Leguminosae sont plus exposés au risque de la surexploitation dans la mesure où, habituellement, l'alimentation des ruminants en général et des bovins en particulier est assurée par des Poaceae et des Leguminosae. La troisième hypothèse accrédite les recherches antérieures au Bénin qui ont montré que les usages des ressources naturelles varient en fonction des groupes sociolinguistiques, du sexe et de l'âge.

\section{MATÉRIEL ET MÉTHODES}

\subsection{Milieu d'étude}

Cette étude a été menée dans la zone d'influence des forêts classées de l'Ouémé Supérieur, des MontsKouffé, Wari-Maro (Figure 1) qui sont les forêts les plus importantes dans la région centrale du Bénin (378 542 ha). Située dans les départements du Borgou, de la Donga et des Collines (2461258 habitants selon INSAE, 2013), cette zone est localisée entre les latitudes $8^{\circ} 80^{\prime}$ et $9^{\circ} 47^{\prime}$ Nord et entre les longitudes $1^{\circ} 55^{\prime}$ et $2^{\circ} 30^{\prime}$ Est, et est incluse dans la zone de transition soudano-guinéenne sensu White (1983). Elle est caractérisée par deux saisons dont une pluvieuse de mai à octobre (1247 mm par an en moyenne) et une

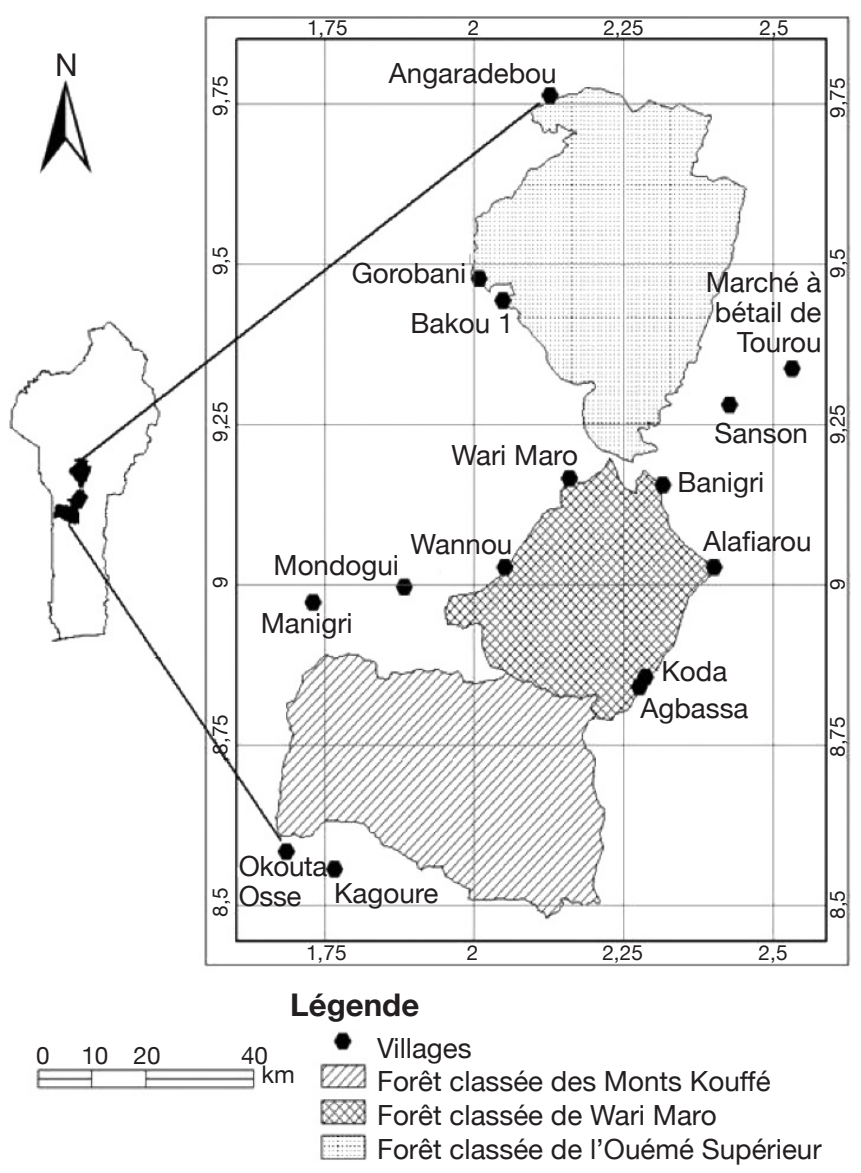

Figure 1. Localisation des villages de l'étude autour des trois forêts classées de la zone soudano-guinéenne du Bénin - Localization of the studied villages around the three forests reserves in the Sudano-Guinean zone of Benin. 
sèche étalée sur environ six mois (novembre à avril). Les températures moyennes annuelles sont de l'ordre de 26 à $27^{\circ} \mathrm{C}$ avec des valeurs extrêmes pouvant aller de $21^{\circ} \mathrm{C}$ (décembre-janvier) à $40^{\circ} \mathrm{C}$ (février-avril) et une humidité relative basse (10 à $40 \%$ de décembre à janvier) à forte (85 à $98 \%$ de juillet à aout).

La végétation naturelle est constituée de forêts galeries, savanes boisées, savanes arborées, savanes arbustives et de végétation saxicole généralement établies sur des sols ferrallitiques et ferrugineux tropicaux. Pendant la saison sèche, la région d'étude accueille des troupeaux transhumants du nord-Bénin, du Nigeria, du Niger et du Burkina Faso qui exploitent, avec les troupeaux sédentaires, les ressources fourragères de la région (Teka et al., 2007). La longue durée de la saison sèche est un facteur limitant la disponibilité du fourrage herbager dans ladite zone. Les éleveurs ont alors recours aux ligneux fourragers qui disposent de feuillage vert en cette période. Les essences comme Khaya senegalensis, Pterocarpus erinaceus et Afzelia africana sont souvent sollicitées mais n'en demeurent pas les seules.

\section{2. Échantillonnage et collecte des données}

Deux types principaux de données ont été utilisés : les données primaires (issues des enquêtes) et les données secondaires (issues de la littérature). Les données primaires ont été collectées au moyen d'enquêtes ethnobotaniques. En prélude à la collecte de données primaires, un sondage préliminaire a été fait au niveau de 60 personnes choisies au hasard dans la population pour savoir si elles utilisent des ligneux fourragers. Pour cette première phase, la proportion $p$ (\%) des personnes utilisant des ligneux fourragers a été estimée à $83,33 \%$. Un seuil de troncature fixé à $\geq 25$ ans d'âge a été intentionnellement appliqué lors de l'échantillonnage des enquêtés pour ne prendre en compte que des personnes ayant déjà une certaine expérience pastorale en termes d'utilisation des ligneux fourragers dans l'alimentation des animaux. La taille de l'échantillon des enquêtés, calculée en utilisant la formule de Dagnelie (1998) donnant 214,45, a été arrondie à 220 :

$$
\mathrm{N}=\frac{U_{1-\alpha / 2}^{2} \times p(1-p)}{d^{2}}
$$

où $\mathrm{N}=$ taille de l'échantillon; $U_{1-\alpha / 2}^{2}$ est la valeur de la loi normale pour un risque $\alpha=0,05 ; U_{1-\alpha / 2}=1,96 ; p=$ $50 / 60=0,8333$ est la proportion de personnes enquêtées connaissant ou utilisant des ligneux fourragers; $d$ est l'erreur marginale fixée à 0,05 . Les enquêtés ont été répartis par groupe socioéconomique dont les caractéristiques sont résumées dans le tableau 1.

Les entretiens se sont focalisés essentiellement sur : - les espèces de ligneux fourragers utilisées dans l'alimentation des animaux ;

- les lieux de récolte des ligneux fourragers ;

Tableau 1. Effectifs et caractéristiques des enquêtés - Number and characteristics of interviewed people.

\begin{tabular}{|c|c|c|c|c|}
\hline \multirow[t]{2}{*}{ Catégories } & \multicolumn{4}{|c|}{ Groupe sociolinguistique } \\
\hline & Bariba & Nago & Peul & Total \\
\hline $\begin{array}{r}\text { Âge moyen des hommes : jeunes } \\
\text { adultes } \\
\text { vieux }\end{array}$ & $\begin{array}{l}32,33 \pm 3,41 \\
41 \pm 3,41 \\
65,82 \pm 11,27\end{array}$ & $\begin{array}{l}28,87 \pm 3,94 \\
41,63 \pm 4,58 \\
58,18 \pm 6,14\end{array}$ & $\begin{array}{l}27,12 \pm 2,88 \\
41,34 \pm 4,41 \\
59,02 \pm 7,97\end{array}$ & $\begin{array}{l}28,10 \pm 4,85 \\
41,12 \pm 4,12 \\
60,58 \pm 9,07\end{array}$ \\
\hline $\begin{array}{r}\text { Âge moyen des femmes : jeunes } \\
\text { adultes } \\
\text { vieilles }\end{array}$ & $\begin{array}{l}33,44 \pm 2,65 \\
45 \pm 4,08 \\
62 \pm 9,09\end{array}$ & $\begin{array}{l}27,40 \pm 2,39 \\
39,94 \pm 4,97 \\
59,86 \pm 6,72\end{array}$ & $\begin{array}{l}- \\
- \\
-\end{array}$ & $\begin{array}{l}30,26 \pm 5,22 \\
40,82 \pm 5,13 \\
28,10 \pm 4,85\end{array}$ \\
\hline $\begin{array}{r}\text { Hommes : jeunes } \\
\text { adultes } \\
\text { vieux }\end{array}$ & $\begin{array}{r}6 \\
17 \\
17\end{array}$ & $\begin{array}{r}8 \\
11 \\
11\end{array}$ & $\begin{array}{l}16 \\
31 \\
37\end{array}$ & $\begin{array}{l}30 \\
59 \\
65\end{array}$ \\
\hline $\begin{array}{r}\text { Femmes : jeunes } \\
\text { adultes } \\
\text { vieilles }\end{array}$ & $\begin{array}{l}9 \\
9 \\
5\end{array}$ & $\begin{array}{l}10 \\
18 \\
15\end{array}$ & $\begin{array}{l}- \\
- \\
-\end{array}$ & $\begin{array}{l}19 \\
27 \\
20\end{array}$ \\
\hline $\begin{aligned} \text { Cheptel moyen /enquêté } & \begin{array}{r}\text { caprins } \\
\text { ovins } \\
\text { bovins }\end{array}\end{aligned}$ & $\begin{array}{l}4,94 \pm 3,67 \\
3,91 \pm 4,24 \\
0,18 \pm 0,73\end{array}$ & $\begin{array}{l}7,48 \pm 8,32 \\
2,37 \pm 4,39 \\
0,13 \pm 1,17\end{array}$ & $\begin{array}{c}3,27 \pm 5,85 \\
29,18 \pm 35,68 \\
100,57 \pm 161,22\end{array}$ & $\begin{array}{c}5,14 \pm 6,59 \\
13,50 \pm 26,11 \\
40,51 \pm 113,17\end{array}$ \\
\hline $\begin{aligned} \text { Niveau d'instruction : } & \text { non instruit } \\
& \text { primaire } \\
& \text { secondaire } \\
& \text { alphabétisé }\end{aligned}$ & $\begin{array}{r}55 \\
3 \\
0 \\
0\end{array}$ & $\begin{array}{l}57 \\
5 \text { dont } 1 \text { femme } \\
12 \text { dont } 3 \text { femmes } \\
0\end{array}$ & $\begin{array}{l}3 \\
0\end{array}$ & $\begin{array}{r}112 \\
8 \\
15 \\
0\end{array}$ \\
\hline
\end{tabular}


- le degré de disponibilité suivant les saisons et dans l'espace ;

- les formes d'exploitation / prélèvement ;

- les fréquences de prélèvement ;

- la préférence des espèces et critères utilisés à cet effet ;

- les raisons de choix des ligneux pâturés ;

- le moment de l'année où l'on a recours au fourrage ligneux ;

- les espèces devenant de plus en plus rares et raisons de raréfaction ;

- les catégories d'animaux (ovins, caprins, bovins) nourris avec le fourrage ligneux.

Le site de la base PROTA4U (http://www. prota4u.org/) a été consulté pour toutes les espèces afin d'avoir des données secondaires sur l'origine, la distribution géographique de ces espèces en Afrique et dans le monde, les différentes formes et domaines d'utilisations, les éventuelles valorisations économiques, etc. Le guide de terrain comportant les noms locaux de la flore du Bénin (de Souza, 2008) a été utilisé pour certaines espèces citées par les populations ainsi que le livre de Arbonnier (2000), Arbres, arbustes et lianes de l'Afrique de l'Ouest. Un herbier a été réalisé pour les espèces non identifiées sur le terrain et a été ramené à l'Herbier National pour leur identification.

Pour déterminer le statut de conservation de chaque espèce, le site de la liste rouge de l'UICN a été utilisé et sur le plan local, le décret d'application $\mathrm{n}^{\circ}$ 96-271 de la loi 93-009 portant régime des forêts en République du Bénin a été consulté ainsi que l'atlas monographique de la biodiversité du Bénin (Adomou et al., 2010 ; Sinsin et al., 2010).

\subsection{Traitement et analyse des données}

Pour identifier les ligneux les plus cités par les populations ainsi que les associations de certains ligneux dans l'alimentation des animaux, une base binaire codée 0 et 1 (espèce citée $=1$ et espèce non citée $=0$ ) a été constituée avec les ligneux fourragers qui ont été cités par $9 \%$ au moins des enquêtés. Cette base a été soumise dans le logiciel $\mathrm{R}$ à une analyse de corrélation graphique (corrgram analysis, [Zuur et al., 2010]). Cette analyse apporte une information complémentaire au calcul des simples taux de citation dans la mesure où deux essences ayant le même taux de citation peuvent subir différentes pressions d'exploitation; le tout dépend des quantités et modes de prélèvement suivant les groupes sociolinguistiques. Une analyse en composantes principales est effectuée sur le tableau croisé des données liées aux groupes sociolinguistiques et les espèces les plus citées afin d'apprécier une éventuelle préférence des groupes sociolinguistiques pour certaines catégories de ligneux fourragers.

Pour identifier les ligneux prioritaires pour le pastoralisme et pour la conservation, une liste préliminaire a été réalisée à partir des données d'enquêtes sur ces ligneux fourragers. L'ordre de priorité de ces ligneux pour le pastoralisme a été déterminé par les populations au travers des entretiens de groupe associés à leur taux de citation. La priorisation pour la conservation a été faite sur la base d'un certain nombre de critères, à savoir :

- l'origine des espèces (autochtone, allochtone, non connue) ;

- la disponibilité de l'espèce ;

- les types biologiques ;

- la distribution de l'espèce dans la zone ;

- les menaces qui pèsent sur l'espèce (formes et intensité de prélèvement - très prélevé moyennement prélevé - rarement prélevé, etc.) ;

- la législation sur l'espèce (protégée ou non);

- le statut UICN de l'espèce ;

- la valeur économique des ligneux fourragers ;

- la valeur fourragère et l'appétibilité de l'espèce.

Ces critères de priorisation sont inspirés de ceux définis par Brehm et al. (2010), modifiés par Idohou et al. (2013). La classification des ligneux a été faite suivant quatre méthodes et ceci de façon indépendante (Brehm et al., 2010) avec le rang Ri de chacune des vingt premières espèces prioritaires pour la conservation suivant chaque procédure. Il s'agit de la procédure des Points Scores (PSP : Point Scoring Procedure); la procédure des Points Scores Pondérés (PSPW : Point Scoring Procedure with Weighting); la procédure basée sur un Système de Rangs Composés (CRS : Compound Ranking System) et la procédure basée sur un Système de Rang Binomial (BRS: Binomial Ranking System). Ces différentes procédures de traitement sont appliquées sur l'ensemble des critères choisis (Figure 2). Ensuite, il a été procédé à un croisement des différents résultats de priorisation afin de ressortir une liste définitive de priorité des ligneux pour la conservation dans les terres de parcours de la zone soudano-guinéenne du Bénin. Ceci a été réalisé sur la base des scores (représentant le nombre de procédures ayant classé l'espèce) et les scores indexés obtenus en faisant la somme des produits des rangs par leur indice (ou somme des rangs occupés par l'essence). Le choix des quatre procédures pour la priorisation des ligneux fourragers est lié à la complexité des appréciations des différentes formes d'utilisation (pastorale ou non) et celles relatives à leur conservation. Ainsi, plusieurs facteurs sont pris en compte pour définir leur priorité de conservation en fonction de leur valeur d'usage. 


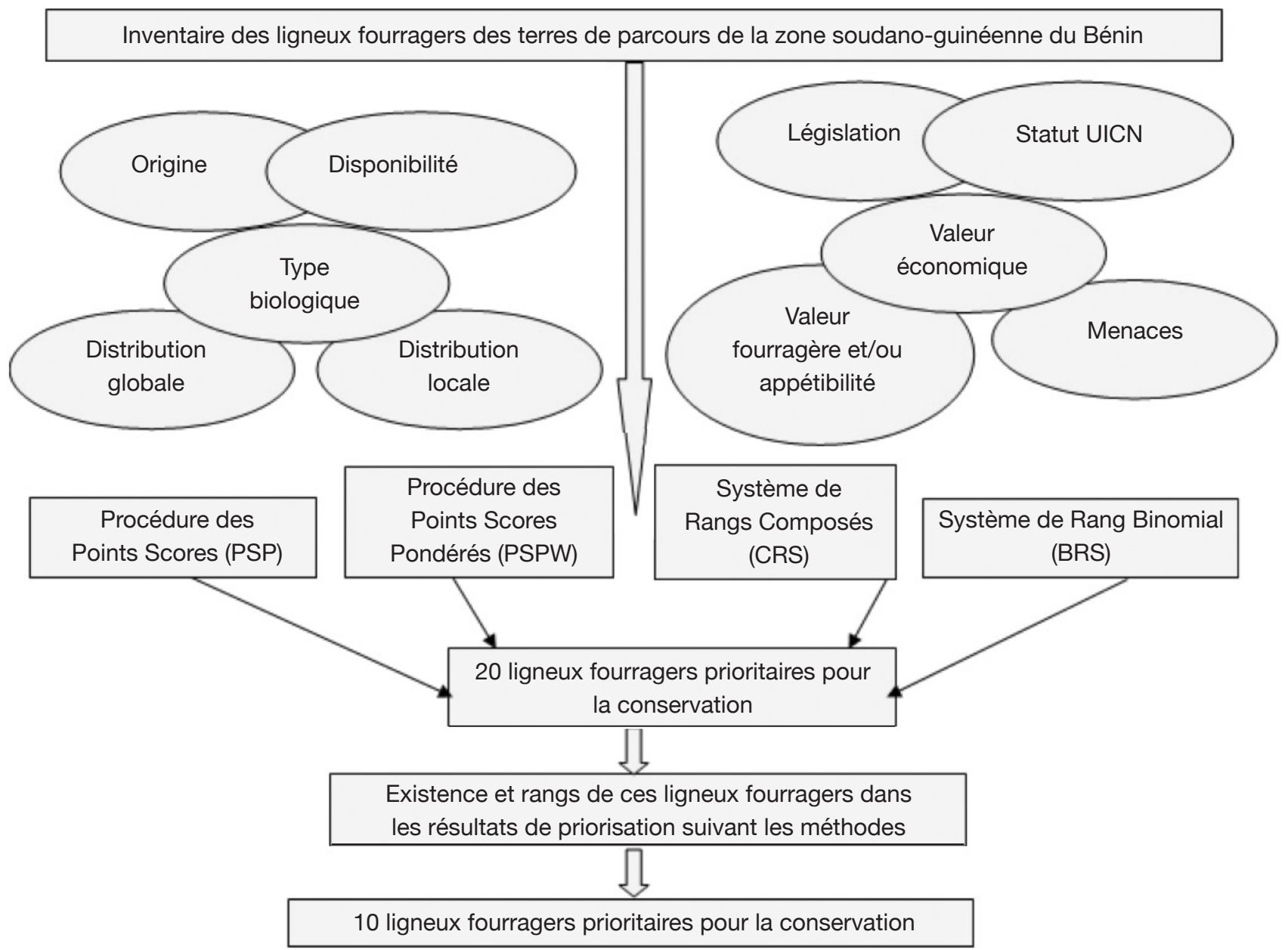

Figure 2. Procédure méthodologique de priorisation des ligneux fourragers pour la conservation dans les terres de parcours de la zone soudano-guinéenne du Bénin (adapté de Brehm et al., 2010) - Methodological procedure for prioritization of fodder trees for conservation in the Sudano-Guinean zone of Benin (adapted from Brehm et al., 2010).

\section{RÉSULTATS}

\subsection{Diversité en familles, genres et espèces des ligneux fourragers}

Au total, 48 ligneux fourragers répartis en 19 familles et 37 genres ont été inventoriés. Les familles les plus représentées sont les Leguminosae $(27,1 \%)$, les Moraceae (16,7 \%), les Rubiaceae et les Combretaceae (8,3\% chacune) et les Anacardiaceae $(6,2 \%)$. Le genre Ficus est le plus représenté $(16,7 \%)$, suivi de Acacia $(6,2 \%)$, Combretum et Gardenia représentant chacun $4,2 \%$ des espèces et les autres genres constitués chacun d'une espèce, soit 2,1\% (Figure 3). La plupart de ces ligneux fourragers sont allochtones (36 soit $75 \%$ ), contre seulement 12 autochtones (25\%).

\subsection{Importance pastorale des ligneux fourragers}

L'analyse de la figure 4 montre que les espèces les plus citées par les populations (plus de $70 \%$ des enquêtés) comme faisant l'objet d'utilisation pastorale sont Pterocarpus erinaceus, Afzelia africana, Khaya senegalensis. D'autres espèces telles que Ficus sycomorus, Daniellia oliveri, Ficus thonningii et Mangifera indica sont relativement moins citées (moins de $35 \%$ ). Par ailleurs, $70 \%$ des enquêtés exploitent le P.erinaceus en combinaison avec A.africana et/ ou K. senegalensis. D'autres espèces sont rarement utilisées en association ( $M$. indica et $F$. sycomorus) ou utilisées seules (M. indica et Gmelina arborea, Ficus umbellata et Vitellaria paradoxa).

Les plus faibles fréquences de citations ont été obtenues pour des espèces comme Acacia ataxacantha, Balanites aegyptiaca, Bombax costatum, Ficus lutea, Ficus polita, Ficus sur, Parkia biglobosa, Psidium guajava, Vitex doniana. Toutes les autres espèces, en dehors de celles prises en compte par la figure 4, ont été citées par moins de $6 \%$ des enquêtés.

Les douze ligneux fourragers les plus prioritaires pour l'alimentation des animaux sont P.erinaceus, A.africana, K. senegalensis, M.indica, Ficus 


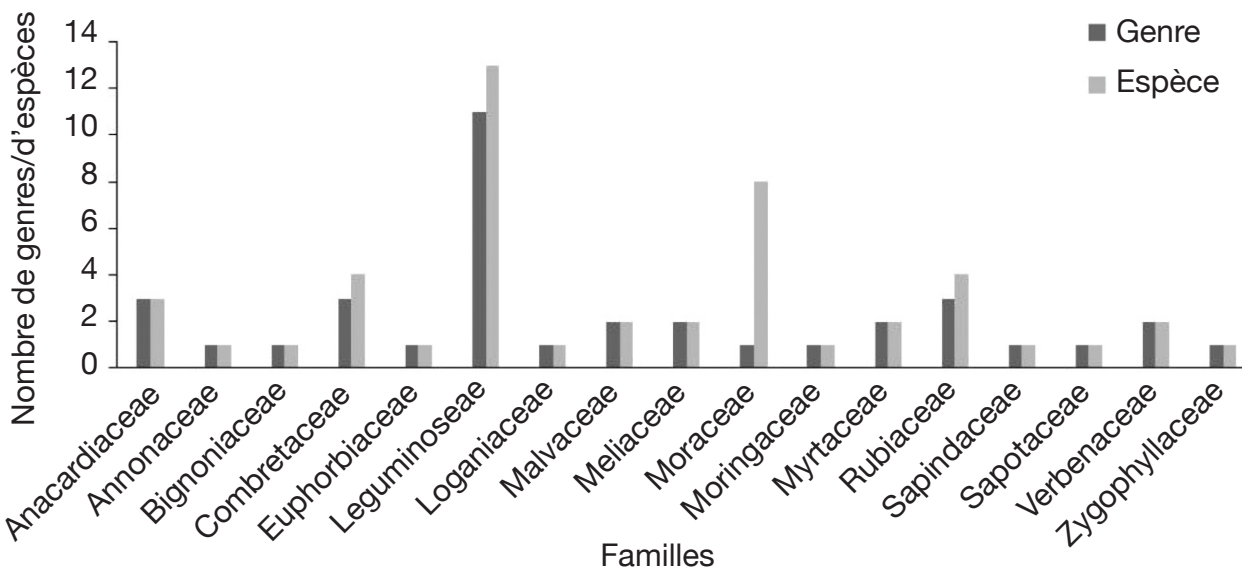

Figure 3. Répartition des genres et espèces des ligneux fourragers au sein des familles Distribution of genus and fodder tree species within families.

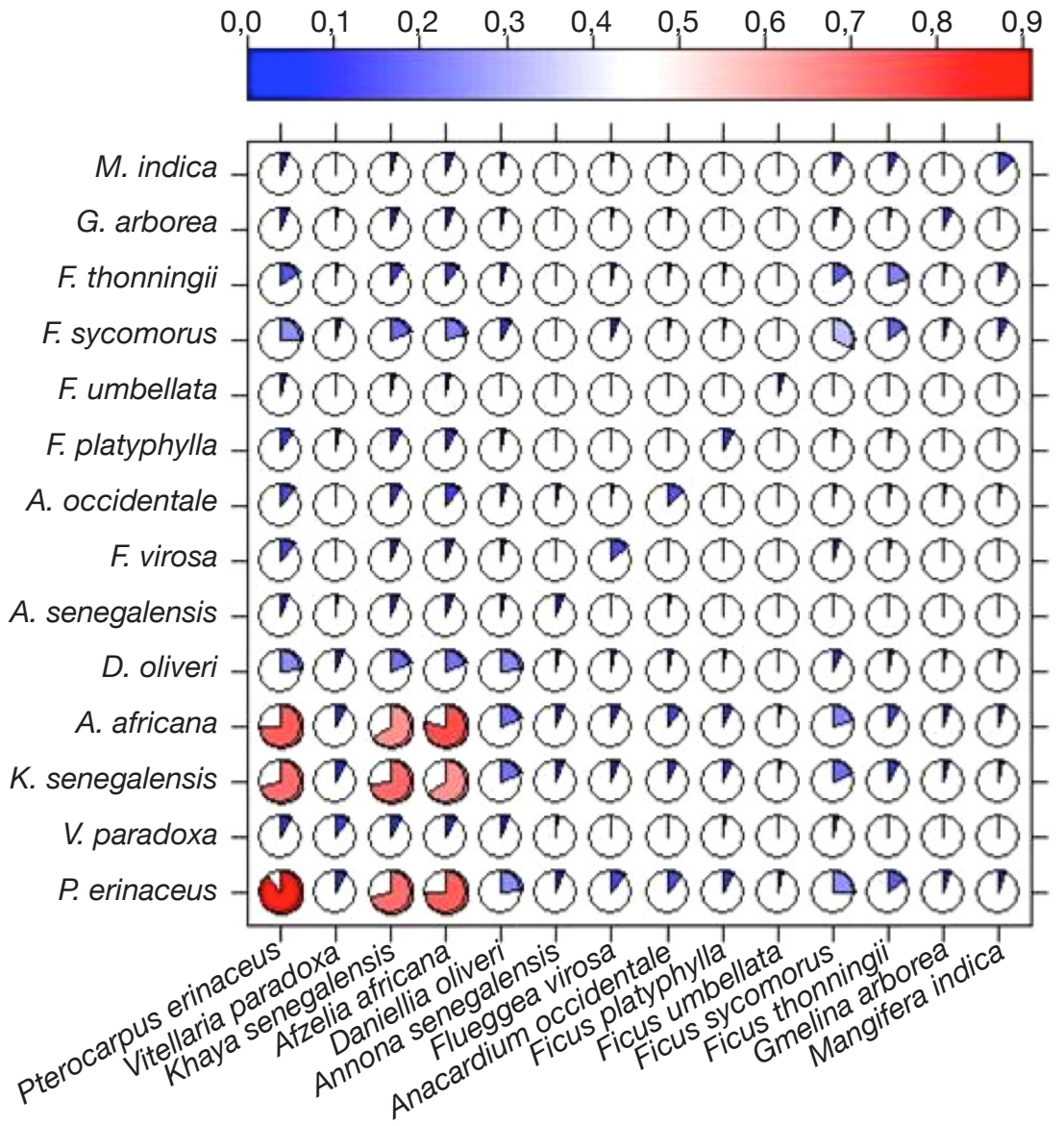

Figure 4. Corrgram montrant la fréquence d'utilisation combinée des ligneux fourragers pris 2 à 2 - Corrgram showing the use frequency of pairs of fodder trees.

platyphylla, D. oliveri, F. umbellata, Flueggea virosa, Annona senegalensis, F. thonningii, G. arborea et $V$. paradoxa (Tableau 2). Les ligneux fourragers prioritaires pour le pastoralisme sont prédominés par les Leguminosae et les Moraceae (25\% chacun).
Sur les six premières espèces prioritaires pour le pastoralisme, trois sont de la famille des Leguminosae.

\subsection{Exploitation des ligneux fourragers par les différents groupes sociolinguistiques}

Il est noté que $80 \%$ des Nagos et $63 \%$ des Baribas enquêtés coupent moins de $25 \%$ de la masse foliaire des ligneux exploités, alors que seulement $2 \%$ des Peuls appliquent ce niveau d'intensité de coupe (Figure 5). Aucun Bariba ni Nago n'exploite au-delà de $75 \%$ de la masse foliaire, par contre $41 \%$ des Peuls affirment qu'ils exploitent l'intégralité de la masse foliaire et $45 \%$ appliquent une intensité de $75 \%$ de coupe.

Les critères de choix des ligneux varient suivant les groupes sociolinguistiques. En effet, les critères prioritaires pour le choix des ligneux fourragers sont respectivement: 1'appétibilité pour les Baribas (55\%), la disponibilité pour les Nagos $(50 \%)$ des espèces et leur valeur alimentaire (au niveau des animaux) pour les Peuls $(47 \%)$. $\mathrm{Si}$ le critère de la productivité a peu d'importance pour les Nagos, les autres critères présentent une importance relative pour l'ensemble des groupes sociolinguistiques dont les proportions varient entre 12 et $23 \%$ des enquêtés (Figure 6).

L'analyse en composantes principales, effectuée sur les données liées aux groupes sociolinguistiques utilisant différents types de ligneux fourragers, montre que l'ensemble des trois premiers axes expliquent environ $75 \%$ de l'information initiale, ce qui est suffisant pour garantir une précision d'interprétation. Le tableau 3 montre les coefficients de corrélation entre chacun des trois axes et les ligneux.

L'analyse de ce tableau montre que :

- l'axe 1 est l'axe d'utilisation de A.africana, $P$. erinaceus, $K$. senegalensis, $M$. indica, D. oliveri, 


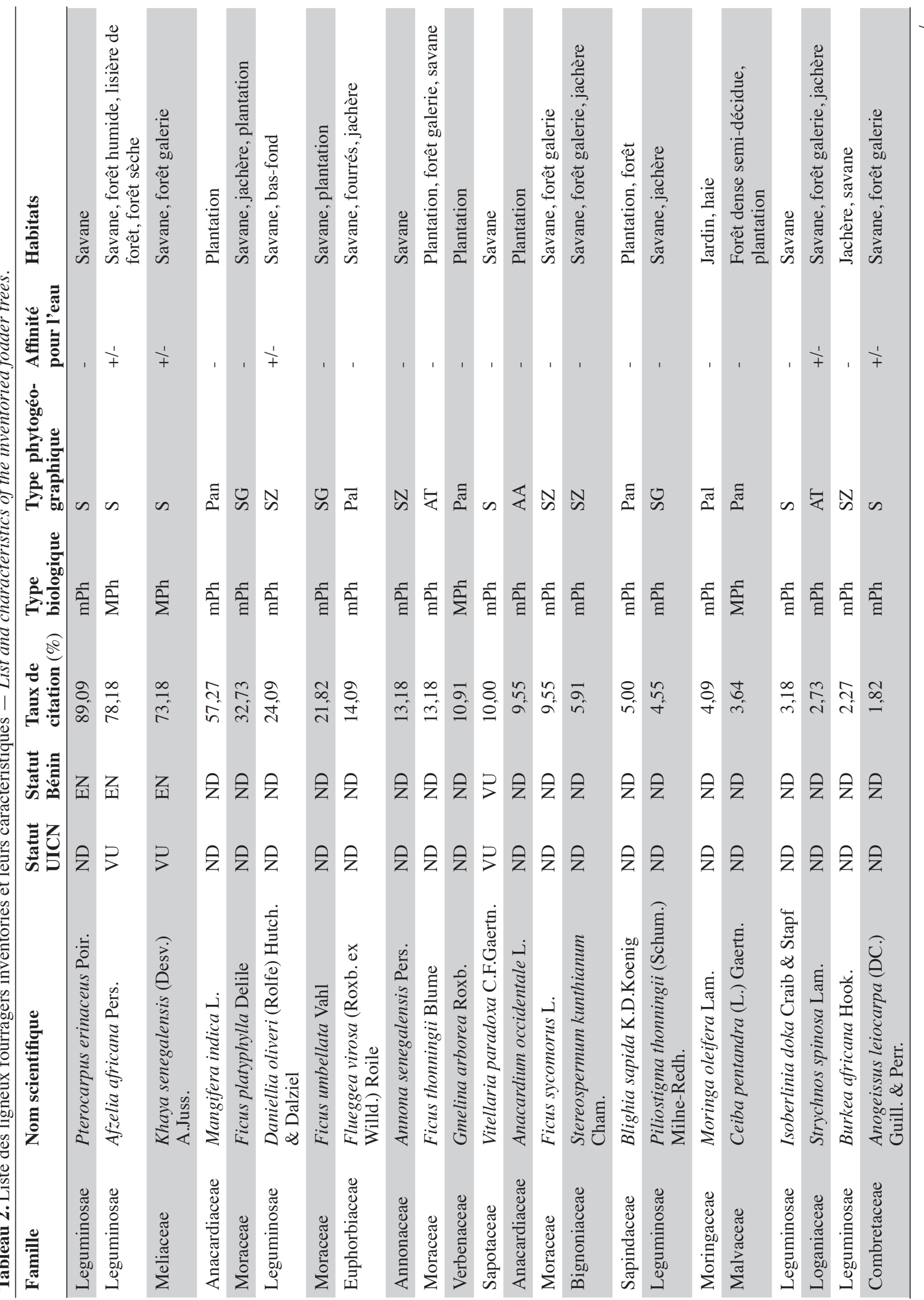




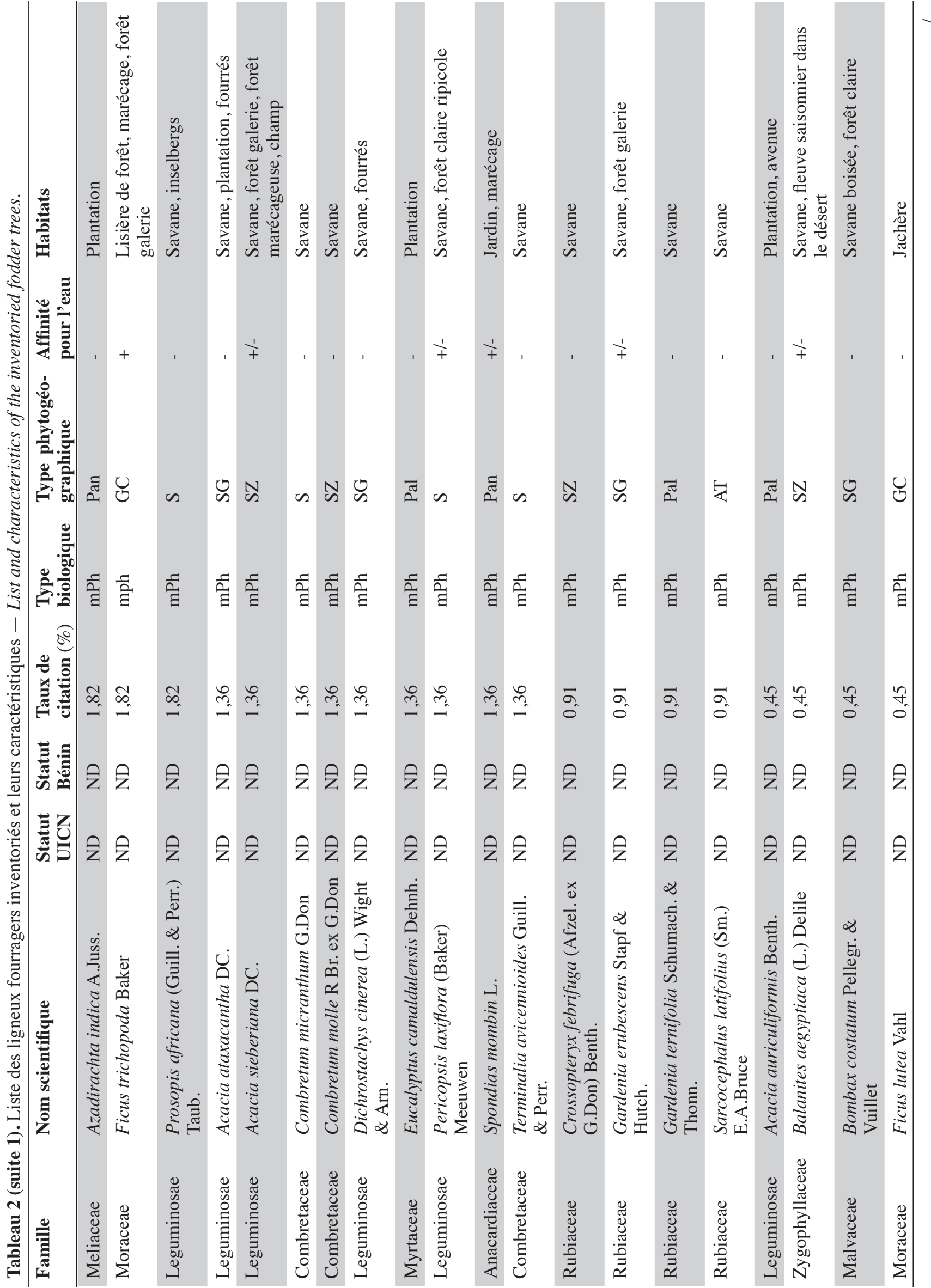



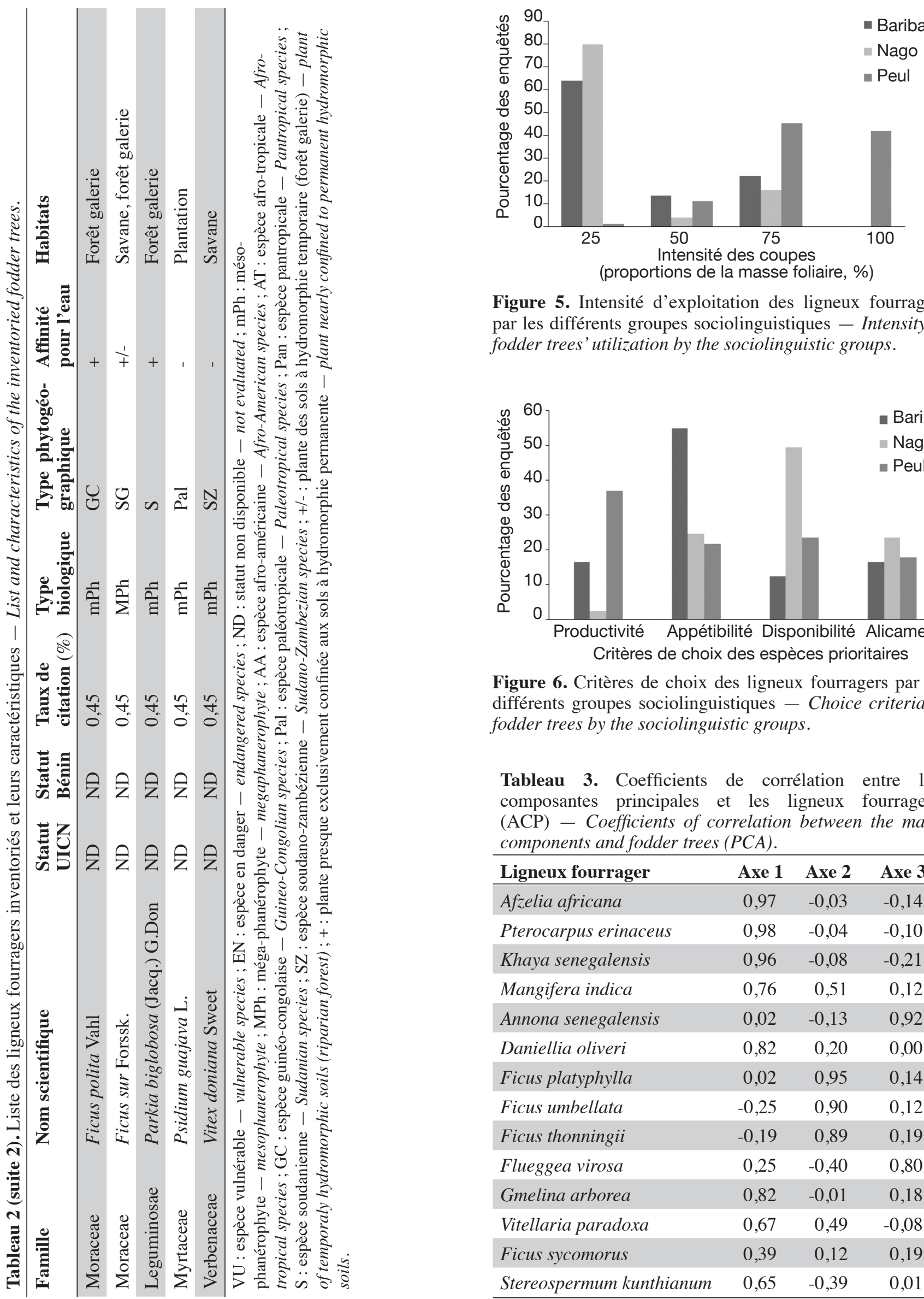

Figure 5. Intensité d'exploitation des ligneux fourragers par les différents groupes sociolinguistiques - Intensity of fodder trees' utilization by the sociolinguistic groups.

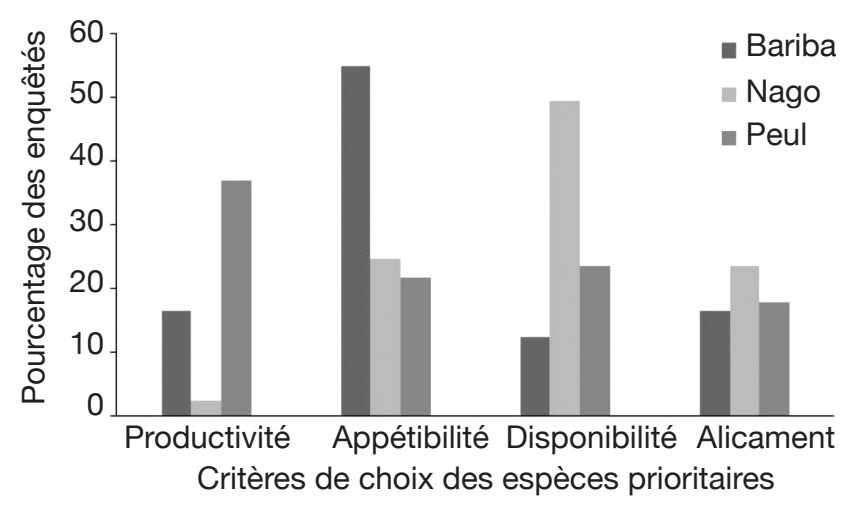

Figure 6. Critères de choix des ligneux fourragers par les différents groupes sociolinguistiques - Choice criteria of fodder trees by the sociolinguistic groups.

Tableau 3. Coefficients de corrélation entre les composantes principales et les ligneux fourragers (ACP) - Coefficients of correlation between the main components and fodder trees (PCA).

\begin{tabular}{lccc}
\hline Ligneux fourrager & Axe $\mathbf{1}$ & Axe $\mathbf{2}$ & Axe 3 \\
\hline Afzelia africana & 0,97 & $-0,03$ & $-0,14$ \\
\hline Pterocarpus erinaceus & 0,98 & $-0,04$ & $-0,10$ \\
Khaya senegalensis & 0,96 & $-0,08$ & $-0,21$ \\
\hline Mangifera indica & 0,76 & 0,51 & 0,12 \\
Annona senegalensis & 0,02 & $-0,13$ & 0,92 \\
\hline Daniellia oliveri & 0,82 & 0,20 & 0,00 \\
\hline Ficus platyphylla & 0,02 & 0,95 & 0,14 \\
\hline Ficus umbellata & $-0,25$ & 0,90 & 0,12 \\
Ficus thonningii & $-0,19$ & 0,89 & 0,19 \\
\hline Flueggea virosa & 0,25 & $-0,40$ & 0,80 \\
Gmelina arborea & 0,82 & $-0,01$ & 0,18 \\
Vitellaria paradoxa & 0,67 & 0,49 & $-0,08$ \\
Ficus sycomorus & 0,39 & 0,12 & 0,19 \\
\hline Stereospermum kunthianum & 0,65 & $-0,39$ & 0,01 \\
\hline
\end{tabular}


G.arborea, V.paradoxa et de Stereospermum kunthianum, (coefficients de corrélation $>0,5)$. De cet axe, on note qu'une forte utilisation de A. africana est souvent associée à une utilisation de $P$. erinaceus, $K$. senegalensis, M. indica, D. oliveri, G. arborea, $V$. paradoxa et $S$. kunthianum. Cet axe est formé en dehors de $M$. indica et de $G$. arborea par les espèces savanicoles ;

- l'axe 2 est l'axe d'utilisation de F.platyphylla, $F$. umbellata et F.thonningii. Il est formé majoritairement par des espèces plantées autour des maisons, principalement pour leur ombrage. De cet axe, on peut dire qu'une bonne utilisation de F. platyphylla est souvent associée à une bonne utilisation de $F$. umbellata et $F$. thonningii ;

- l'axe 3 est celui d'utilisation de A. senegalensis et Flueggea virosa, révélant qu'une bonne utilisation de A. senegalensis est souvent associée à une bonne utilisation de $F$. virosa dans l'alimentation des animaux. Cet axe est formé par des espèces de jachères.

La projection des différents ligneux fourragers dans les systèmes d'axes (Figure 7) montre que les Peuls (adultes comme vieux) utilisent beaucoup plus les espèces comme A.africana, P.erinaceus, $K$. senegalensis, M.indica, D.oliveri, G.arborea, $V$. paradoxa et S. kunthianum comme fourrages et

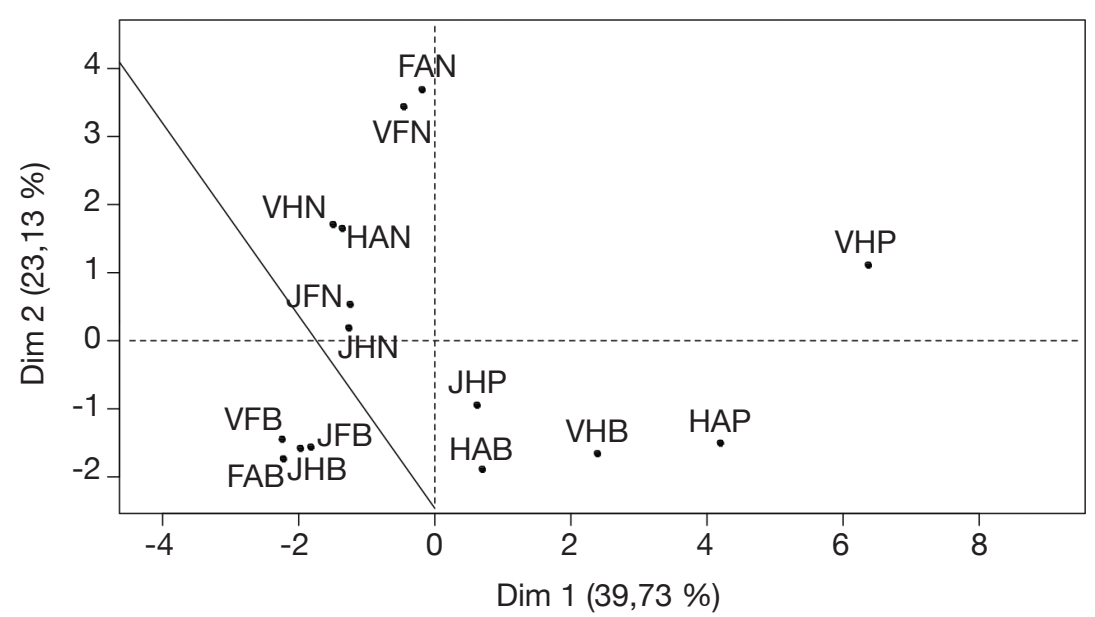

Figure 7. Projection des différents groupes sociolinguistiques dans le système d'axes 1 et 2 - Projection of the sociolinguistic groups in axes 1 and 2 .

HAB : homme adulte Bariba - adult man Bariba ; FAB : femme adulte Bariba - adult woman Bariba; JHB : jeune homme Bariba - young man Bariba; JFB : jeune femme Bariba - young woman Bariba; VHB : vieil homme Bariba - old man Bariba; VFB : vieille femme Bariba - old woman Bariba; HAN : homme adulte Nago - adult man Nago; FAN : femme adulte Nago - adult woman Nago ; JHN : jeune homme Nago - young man Nago ; JFN : jeune femme Nago - young woman Nago ; VHN : vieil homme Nago - old man Nago; VFN : vieille femme Nago - old woman Nago ; HAP : homme adulte Peul - adult man Peul; JHP : jeune homme Peul - young man Peul; VHP : vieil homme Peul — old man Peul. presque pas du tout les espèces du genre Ficus. Par contre, on note de cette figure que les espèces du genre Ficus et M. indica sont très utilisées par les Nagos, quels que soient leur âge et leur sexe.

Par ailleurs, la figure $\mathbf{8}$ montre que les fourrages de A. senegalensis et de F. virosa sont plus utilisés par les femmes adultes Nagos, les hommes adultes Baribas et les vieux hommes Baribas. Les autres groupes sociolinguistiques utilisent très faiblement ces

\subsection{Priorisation pour la conservation des ligneux}

Les différentes méthodes de classification des ligneux fourragers en espèces prioritaires pour la conservation ont conduit à l'identification de 28 espèces par une ou plusieurs de ces méthodes (Tableau 4). Elles sont regroupées en 13 familles et 24 genres (Figure 9), les Leguminosae sont les plus représentées en genres (37,5\% chacun) et en espèces $(32,1 \%$ chacun). La majorité des familles $(60 \%)$ sont représentées chacune par une espèce. La famille des Moraceae est peu diversifiée en genre avec ses quatre espèces prioritaires ppartenant toutes au genre Ficus.

Afzelia africana a été identifiée comme première essence ligneuse fourragère prioritaire pour la conservation, suivant chacune des quatre procédures. Elle est suivie de P.erinaceus, K. senegalensis, $\quad$ V.paradoxa, D. oliveri, B. aegyptiaca, Piliostigma thonningii, F. virosa, P. biglobosa et Prosopis africana, formant ainsi la liste des dix ligneux fourragers prioritaires pour la conservation. Les ligneux comme Bombax costatum, Acacia auriculiformis, Dichrostachys cinerea, Moringa oleifera et Sarcocephalus latifolius, qui n'ont été classés prioritaires que par une seule procédure et dans des rangs relativement plus élevés, sont donc d'ordre de priorité moindre pour la conservation.

\section{DISCUSSION}

\subsection{Diversité des ligneux fourragers}

Les ligneux fourragers constituent le principal recours des éleveurs pour l'alimentation du bétail en période sèche du fait que l'alimentation des animaux est essentiellement basée sur les pâturages naturels à la fin de la 


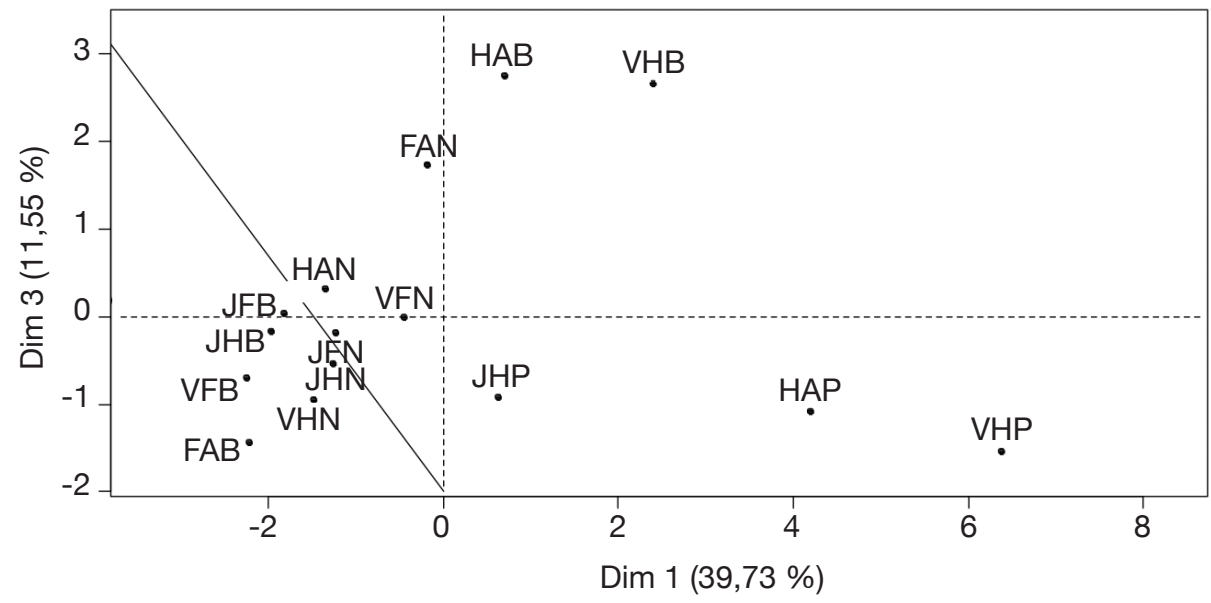

Figure 8. Projection des différents groupes sociolinguistiques dans le système d'axes 1 et 3 - Projection of the sociolinguistic groups in axes 1 and 3.

Explication des groupes - meaning of the groups : voir figure 7 - see figure 7.

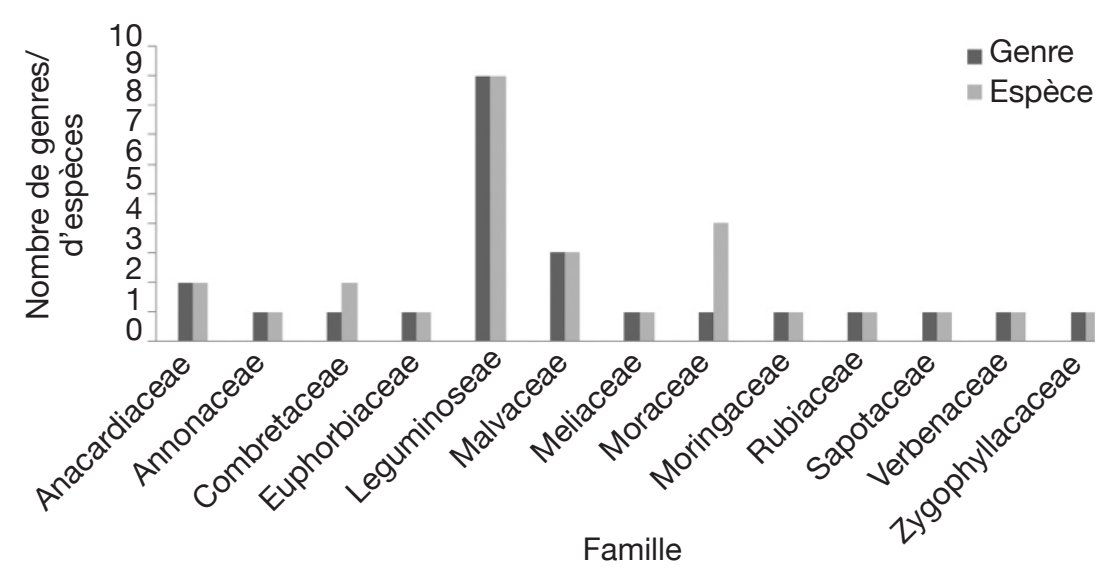

Figure 9. Répartition, par famille, des genres et espèces des ligneux prioritaires pour la conservation - Distribution of genus and fodder tree species prioritized for conservation within families.

période d'exploitation des résidus de récolte et parfois de la paille quasiment sèche. L'hypothèse suivant laquelle les éleveurs feraient plus recours aux ligneux fourragers ayant des affinités pour les milieux humides, qui de ce fait subiront plus de pression pastorale que d'autres, est rejetée. En effet, au niveau des 48 ligneux fourragers inventoriés, 14 soit $29,2 \%$ et seulement 3 ligneux fourragers sur les 12 classés prioritaires pour le pastoralisme $(25 \%)$ présentent une certaine affinité pour les milieux humides. Dès lors, les ligneux fourragers exploités pour alimenter les ruminants en saison sèche ne sont pas majoritairement des espèces inféodées aux milieux humides. Les énormes besoins en fourrage aérien obligent les éleveurs et leurs troupeaux à parcourir de grandes distances à travers tous les milieux (savane, champs, jachère, forêt galerie, zone de bas-fond). La richesse spécifique (48 espèces) des ligneux fourragers (Tableau 4) exploités par les populations riveraines des trois forêts représente 1,71\% de la flore du Bénin estimée à 2807 espèces (Akoègninou et al., 2006 ; Adomou, 2010). La forte prédominance des Leguminoseae observée dans cette étude pourrait être expliquée par le fait que l'élément de base de la flore locale est naturellement prédominée par cette famille (White, 1983). Ces résultats confirment une forte utilisation de la végétation naturelle pour l'alimentation du bétail (Sarr et al., 2013).

En comparaison à des études précédentes, la diversité floristique des ligneux fourragers dans le milieu d'étude semble relativement faible (59 dans l'État de Katsina au Nigeria [Tukur et al., 2013]; 60 dans les savanes du Nord-Cameroun [Onana, 1995] ; 67 dans les agrosystèmes de la région désertique de Himachal Pradesh de l'Himalaya [Rawat et al., 2011]; 68 dans la région du Poro au nord de la Côte d'Ivoire [Silue et al., 2014]). Elle est par contre plus élevée que les 18 ligneux fourragers dénombrés dans la vallée du Rift en Éthiopie (Shenkute et al., 2012) et les 28 recensés dans la région de Wolayta au sud de l'Éthiopie (Geta et al., 2014). Plusieurs facteurs peuvent expliquer ces différences, dont notamment les variations des conditions pédoclimatiques et leurs influences sur la distribution spatiale et géographique des flores (Adomou et al., 2006).

Parmi les espèces fourragères prioritaires identifiées, aucune n'est endémique au Bénin. Certaines comme $M$. indica, D. oliveri, F. virosa et A. senegalensis sont moins citées dans la littérature comme faisant l'objet de pâturage, alors que d'autres comme $P$.erinaceus, $K$. senegalensis et A. africana sont pour la plupart exploitées dans d'autres pays ; au Sénégal (Sarr et al., 2013), au Tchad (Bechir et al., 2009 ; Bechir et al., 2012), au Burkina Faso (Hahn-Hadjali et al., 2000), au Cameroun (Onana, 1995 ; Onana et al., 2002) et en Côte d'Ivoire (Silue et al., 2014). Les critères de disponibilité et surtout 
Tableau 4. Liste de 28 espèces ligneuses fourragères prioritaires pour la conservation - List of 28 priority fodder trees for conservation.

\begin{tabular}{|c|c|c|c|c|c|c|}
\hline Espèce & PSP & PSPW & CRS & BRS & Score & Score indexé \\
\hline Afzelia africana Pers. & $\mathrm{R} 1$ & $\mathrm{R} 1$ & $\mathrm{R} 1$ & $\mathrm{R} 1$ & 4 & 4 \\
\hline Pterocarpus erinaceus Poir. & $\mathrm{R} 2$ & $\mathrm{R} 2$ & $\mathrm{R} 2$ & $\mathrm{R} 2$ & 4 & 11 \\
\hline Khaya senegalensis (Desv.) A.Juss. & R3 & R3 & R3 & $\mathrm{R} 2$ & 4 & 13 \\
\hline Vitellaria paradoxa C.F.Gaertn. & R9 & R5 & R5 & $\mathrm{R} 2$ & 4 & 22 \\
\hline Daniellia oliveri (Rolfe) Hutch. \& Dalziel & $\mathrm{R} 13$ & $\mathrm{R} 10$ & $\mathrm{R} 12$ & $\mathrm{R} 2$ & 4 & 37 \\
\hline Balanites aegyptiaca $($ L.) Delile & R4 & R7 & $\mathrm{R} 18$ & $\mathrm{R} 10$ & 4 & 39 \\
\hline Piliostigma thonningii (Schumach.) Milne-Redh. & $\mathrm{R} 13$ & $\mathrm{R} 15$ & $\mathrm{R} 8$ & $\mathrm{R} 10$ & 4 & 46 \\
\hline Flueggea virosa (Roxb. ex Willd.) Voigt & $\mathrm{R} 17$ & $\mathrm{R} 17$ & R6 & R9 & 4 & 49 \\
\hline Parkia biglobosa (Jacq.) R. Br. ex G.Don & $\mathrm{R} 17$ & $\mathrm{R} 19$ & $\mathrm{R} 8$ & R6 & 4 & 50 \\
\hline Prosopis africana (Guill. \& Perr.) Taub. & R17 & R19 & $\mathrm{R} 14$ & $\mathrm{R} 7$ & 4 & 57 \\
\hline Ficus thonningii Blume & R17 & $\mathrm{R} 17$ & R14 & $\mathrm{R} 10$ & 4 & 58 \\
\hline Mangifera indica $\mathrm{L}$. & $\mathrm{R} 4$ & $\mathrm{R} 4$ & $\mathrm{R} 4$ & - & 3 & 12 \\
\hline Ficus platyphylla Delile & $\mathrm{R} 4$ & R5 & R7 & - & 3 & 16 \\
\hline Annona senegalensis Pers. & $\mathrm{R} 4$ & $\mathrm{R} 8$ & - & $\mathrm{R} 10$ & 3 & 22 \\
\hline Combretum micranthum R. Br. ex G.Don & $\mathrm{R} 10$ & $\mathrm{R} 12$ & - & $\mathrm{R} 10$ & 3 & 32 \\
\hline Vitex doniana Sweet & $\mathrm{R} 10$ & $\mathrm{R} 12$ & - & $\mathrm{R} 10$ & 3 & 32 \\
\hline Azadirachta indica A.Juss. & $\mathrm{R} 13$ & $\mathrm{R} 12$ & $\mathrm{R} 12$ & - & 3 & 37 \\
\hline Ficus sycomorus L. & $\mathrm{R} 10$ & $\mathrm{R} 11$ & $\mathrm{R} 18$ & - & 3 & 39 \\
\hline Ceiba pentandra (L.) Gaertn. & $\mathrm{R} 17$ & - & R14 & $\mathrm{R} 10$ & 3 & 41 \\
\hline Ficus umbellata Vahl & $\mathrm{R} 8$ & R9 & - & - & 2 & 17 \\
\hline Anogeissus leiocarpa (DC.) Guill. \& Perr. & - & - & R14 & $\mathrm{R} 10$ & 2 & 24 \\
\hline Isoberlinia doka Craib \& Stapf & - & - & R18 & $\mathrm{R} 8$ & 2 & 26 \\
\hline Anacardium occidentale L. & $\mathrm{R} 13$ & $\mathrm{R} 15$ & - & - & 2 & 28 \\
\hline Sarcocephalus latifolius (Sm.) E. A. Bruce & - & - & $\mathrm{R} 8$ & - & 1 & 8 \\
\hline Dichrostachys cinerea (L.) Wight \& Arn. & - & - & - & $\mathrm{R} 10$ & 1 & 10 \\
\hline Moringa oleifera Lam. & - & - & R11 & - & 1 & 11 \\
\hline Acacia auriculiformis A. Cunn. ex Benth. & - & - & - & R19 & 1 & 19 \\
\hline Bombax costatum Pellegr. \& Vuill. & - & - & - & R19 & 1 & 19 \\
\hline
\end{tabular}

Ri : indique le rang i qu'occupe chacune des vingt premières espèces prioritaires par méthode ; les espèces ayant occupé des rangs ex aequo pour la même procédure portent donc le même indice de rang - Ri indicates the rank $i$ occupied by the first twenty priority species with each method; species with equal rank for the same procedure have the same ranking index; PSP : procédure des Points Scores - Point Scoring Procedure; PSPW : procédure des Points Scores Pondérés - Point Scoring Procedure with Weighting ; CRS : procédure basée sur un Système de Rangs Composés - Compound Ranking System ; BRS : procédure basée sur un Système de Rang Binomial - Binomial Ranking System; Scores : indiquent le nombre de méthodes qui classent l'essence - indicate the number of methods that prioritise the species; Scores indexés : somme des rangs occupés par l'essence si elle est classée par plusieurs méthodes sum of ranking position of each species when classified by several methods.

d'appétibilité expliqueraient les préférences dans les usages pastoraux de ces ligneux.

\subsection{Priorisation des ligneux fourragers}

L'hypothèse qui stipule que les ligneux fourragers de la famille des Leguminosae sont plus utilisés et de facto plus exposés aux risques de la surexploitation a été vérifiée. Sur l'ensemble des ligneux fourragers inventoriés, plus du quart sont de la famille des Leguminosae $(27,1 \%)$ suivis de loin par les 16 autres familles, puisque la seconde famille la mieux représentée est celle des Moraceae (16,7 \%), qui n'est constituée que des Ficus souvent plantés comme arbres 
d'ombrage autour des maisons. Les Ficus ne sont donc exploités que secondairement pour des besoins d'alimentation des petits ruminants par les Nagos et les Baribas, mais en faibles quantités puisqu'ils n'ont pas un grand cheptel (Tableau 1). Par contre, les Peuls, ayant un cheptel plus élevé, ont plus besoin de grandes quantités de fourrages pour alimenter leurs animaux, contrairement aux autres groupes sociolinguistiques qui en font usage, mais à de très faibles proportions. Ceci montre que les Peuls, qui sont des professionnels de l'élevage, surtout du gros bétail, ont plus besoin d'une grande quantité de fourrage pour alimenter leur troupeau et exercent donc la plus forte pression, non pas sur les ligneux plantés autour des habitations, mais sur ceux des parcours naturels. La prédominance des Leguminosae dans cette étude corrobore les travaux de Sanou (2014) dans l'étude du potentiel des ligneux fourragers du terroir de Sokouraba au Bukina Faso. Par contre, Sarr et al. (2013) avaient noté que la famille des Combretaceae était la plus fréquente et la plus importante des ligneux fourragers dans un système agropastoral au Sénégal, suivie de celle des Leguminosae. Le même constat de prédominance des Combretaceae avait été rapporté par Ndiaye et al. (2010) dans les territoires pastoraux au nord Sénégal. Ces différences peuvent s'expliquer par les mêmes variations pédoclimatiques sus-signalées par Adomou et al. (2006) et qui influencent la distribution spatiale et géographique des flores. Dans le Sahel, le pâturage est dominé par les Combretaceae. Les différences sont donc dues à une variation de l'abondance-dominance des espèces dans l'élément de base.

L'hypothèse suivant laquelle l'utilisation des ligneux fourragers varie en fonction des groupes sociolinguistiques, du sexe et de l'âge est vérifiée. En effet, il est noté une spécialisation des groupes sociolinguistiques dans le choix et l'exploitation des ligneux fourragers. La principale raison est liée au milieu de pâturage (les uns pâturent dans la nature alors que les autres pâturent dans les champs et autour des cases). La spécialisation serait aussi liée aux conditions de la récolte, à la disponibilité à proximité ou dans les habitations par rapport aux espèces plantées utilisées en faibles quantités chez les Baribas et les Nagos. Les Peuls, qui, pour alimenter les animaux, coupent entièrement toute la masse foliaire des espèces comme K. senegalensis (Gaoue et al., 2007), A. africana, $P$. erinaceus, $D$. oliveri, réduisent ainsi la capacité de régénération des espèces par défaut de production des semences dans la nature.

Les ligneux en général et les ligneux fourragers, en particulier, n'ont pas les mêmes importances pour les populations rurales. Certains font l'objet d'exploitations plus intenses du fait de leur valeur et des utilités qu'elles en tirent. Dès lors, plusieurs critères ou motivations permettent de les catégoriser.
Hussain et al. (2013) ont procédé à la classification des essences aussi bien herbacées que ligneuses, dans plusieurs catégories, en se basant sur leurs aptitudes à fournir du fourrage dans l'alimentation des animaux. Ainsi, Bauhinia variegata, Morus alba, Quercus dilata ont été identifiées par ces auteurs comme les meilleurs fournisseurs de fourrage aérien dans la région indienne de l'Himalaya. Robertson et al. (2003) ont noté que les résultats de la priorisation des espèces doivent être pris avec beaucoup d'attention dans la mesure où le rang attribué à chaque espèce dépend aussi de l'importance relative des autres espèces sur la base des critères de classification utilisés. En effet, si certaines espèces classées comme étant les plus prioritaires étaient exclues de la liste, d'autres, moins importantes, vont se révéler comme jouant un rôle capital dans l'alimentation des animaux puisqu'elles vont avoir les meilleurs scores. Cela pourrait s'expliquer par l'effet ou le rôle de substitut ou de second choix qu'elles joueraient pour les éleveurs dans l'alimentation de leur cheptel. Cependant, ces espèces peuvent ne pas combler les attentes des éleveurs du fait des effets secondaires que pourrait avoir leur utilisation massive sur la santé ou la productivité du cheptel. Par exemple, certains éleveurs Peuls de la zone d'étude estiment que l'espèce D. oliveri, consommée pendant longtemps durant la saison sèche, causerait plus tard des maladies cutanées comme la galle chez les bovins et parfois des problèmes dans l'articulation des genoux. C'est ce qui fait qu'ils ne l'utilisent que pour un apport d'appoint en l'absence de $K$. senegalensis, de $P$. erinaceus ou de A. africana qui assurent non seulement une bonne santé des animaux (alicament), mais aussi leur confèrent une bonne productivité de lait, surtout au niveau des vaches. Ces résultats corroborent les constats de Silue et al. (2014) qui ont noté que A. africana, P. erinaceus et $K$. senegalensis sont classés comme les trois ligneux fourragers les plus importants avec des taux d'importance respectifs de 93,3 ; 92,8 et $89,5 \%$, au Sénégal.

\subsection{Limites de la méthode de priorisation des ligneux fourragers}

Il existe plusieurs approches méthodologiques de priorisation des espèces (Maxted et al., 2006). L'approche utilisée dans cette étude est celle proposée par Brehm et al. (2010) qui a été déjà appliquée par Idohou et al. (2013) dans l'évaluation des plantes sauvages et leurs cultivars au Bénin. Cette approche est assez flexible dans la mesure où elle permet d'inclure des critères adaptés aux objectifs de la recherche dans le cadre conceptuel des démarches de priorisation. Cette méthodologie est par contre différente de celles utilisées pour établir une liste prioritaire pour la conservation des produits forestiers non ligneux 
au nord Bénin (Vodouhè et al., 2009), au nord est de l'Inde (Saha et al., 2010) et au sud ouest du Cameroun (Egbe et al., 2012). Dans ce dernier cas, par exemple, les populations ont classé, sur la base de la valeur économique d'une part et, d'autre part, sur la base de leur préférence, des essences qu'elles souhaiteraient cultiver ou domestiquer. Dans la présente étude, en dehors des préférences des éleveurs évaluées à travers leur classification, des facteurs, liés aux autres formes d'utilisation au niveau local ou non, ont été prises en compte afin de tenir réellement compte des menaces qui pèsent sur ces essences. Ces menaces ont certainement des effets sur leur conservation et méritent d'être prises en compte dans les perspectives de définition des stratégies de leur gestion rationnelle. En Israël, Barazani et al. (2008) ont procédé à une classification des plantes autochtones, non seulement par ordre de priorité pour la conservation, mais aussi et surtout une catégorisation des espèces par groupe, suivant un gradient de priorité pour la conservation à partir des scores de priorisation. Il ne s'agit pas, dans leur cas, d'une simple liste des espèces suivant leur ordre de priorité. Ce faisant, les stratégies de conservation pourraient cibler directement le groupe le plus prioritaire dans la mesure où les moyens de conservation font souvent défaut. Dans un cas comme dans l'autre, les méthodes de priorisation sont évolutives et les listes des espèces prioritaires devront connaitre des actualisations dans le futur puisque le statut de conservation et des menaces qui pèsent sur les espèces, au niveau local ou international, peuvent varier dans le temps. C'est à ce titre que Brehm et al. (2010) ont souligné que la priorisation des espèces est un processus dynamique. Par exemple, l'introduction d'un ligneux fourrager dans le milieu, par l'aide des bailleurs de fonds, avec la participation des populations, peut entrainer une modification sur la liste de priorité pastorale des ligneux fourragers de la zone.

\subsection{Implications pour une gestion durable des terres de parcours}

Cette étude a permis non seulement d'identifier les ligneux fourragers des terres de parcours en zone soudano-guinéenne du Bénin mais aussi de définir leur ordre de priorité pour le pastoralisme d'une part et pour la conservation d'autre part. Les ligneux fourragers sont des essences à usages multiples. Les petits ruminants et les bovins sont alimentés surtout en saison sèche grâce au fourrage aérien des arbres dans les villages, les champs et les forêts classées de la région d'étude. Ainsi, les critères de choix de ces ligneux et les menaces qui pèsent sur leur utilisation rationnelle sont à prendre en compte dans l'élaboration des activités d'aménagement des terres de parcours afin de bien concilier les besoins d'utilisation des ligneux avec les objectifs de la conservation des ressources génétiques. Toutes les formes d'utilisation des plantes et les facteurs qui influencent leur survie doivent être analysés et conséquemment pris en compte dans la définition des stratégies d'utilisation rationnelle des ligneux fourragers. Ainsi, ils assureront la fonction de survie des animaux tout en satisfaisant les besoins médicinaux, de bois d'œuvre, protection du milieu, etc.

La domestication des espèces prioritaires comme stratégie de conservation par les populations est une approche envisageable (N'Danikou et al., 2011). Dès lors, il faudra conduire des recherches et essais de régénération artificielle des semences des ligneux fourragers. Ceci est d'autant plus nécessaire que les populations reconnaissent elles-mêmes que certaines espèces prioritaires comme A. africana et $K$. senegalensis deviennent de plus en plus rares dans les terres de parcours de la zone d'étude. Ce constat a été fait par Dassou et al. (2014) sur ces mêmes essences au nord Bénin où elles sont fortement utilisées comme plantes médicinales. Des actions de plantation des ligneux fourragers sont donc à promouvoir, surtout ceux prioritaires, en dehors des fruitiers et des arbres d'ombrage. Envisager des plantations privées de ces ligneux fourragers est une solution plausible, mais qui pourrait être limitée par l'exercice du droit de propriété des terres de la région par les éleveurs, surtout Peuls, qui ne sont pas souvent propriétaires terriens.

L'utilisation des ligneux prioritaires pour le pastoralisme dans les activités de plantation, d'afforestation et de regarnissage permettra d'atteindre l'objectif de conservation à travers une exploitation rationnelle de ces ressources.

\section{CONCLUSIONS}

L'utilisation d'une combinaison de méthodes de priorisation a permis d'identifier dans la zone de transition soudano-guinéenne du Bénin une dizaine de ligneux fourragers prioritaires pour la conservation dont Afzelia africana, Pterocarpus erinaceus et Khaya senegalensis. Les essences qui sont exploitées dans les habitations, dans les jachères et dans les forêts classées de la zone d'étude subissent une forte pression de prélèvement du fait de leur caractère d'essence à usages multiples. En matière de pastoralisme, l'appétibilité, la disponibilité des essences et la productivité des animaux qui les consomment sont identifiés par les populations utilisatrices comme les critères de choix des ligneux fourragers. La prise en compte des ligneux fourragers identifiés comme prioritaires pour la conservation dans le choix des essences de reboisement/afforestation permettra d'assurer une utilisation rationnelle des ligneux à usages multiples. 


\section{Remerciements}

Ce travail a été financé par une bourse de thèse doctorale accordée à Clément Sèwadé. Adandé Belarmain Fandohan a été soutenu par la bourse de recherche Georg Forster-Hermes de la Fondation Alexander von Humboldt (fellowship no 3.4-BEN/1155509 STP). Nous adressons nos remerciements à K. Euloge Agbossou, Lucien Imorou, Rodrigue Idohou, Jacob Sèwadé et C.A.M. Sylvestre Djagoun pour leurs multiples soutiens. Nous pensons aussi aux différentes personnes qui nous ont accordé une partie de leur précieux temps lors des enquêtes sur le terrain.

\section{Bibliographie}

Abule E., 2004. Rangeland evaluation in relation to pastoralists' perceptions in the Middle Awash Valley of Ethiopia. PhD thesis: University of the Free State, Bloemfontein (South Africa).

Adomou A.C., 2010. Aperçu sur la flore du Bénin. In: Sinsin B. \& Kampman D., éds. Atlas de la biodiversité de l'Afrique de l'Ouest. Tome 1 : Bénin. Cotonou, Bénin ; Frankfurt/Main, Allemagne : BIOTA, 144-150.

Adomou A.C., Sinsin B. \& van der Maesen L.J.G., 2006. Phytosociological and chorological approaches to phytogeography: a study at meso-scale in Benin. Syst. Geogr. Pl., 76, 155-178.

Adomou A.C., Sinsin B., Akoégninou A.A. \& van der Maesen L.J.G., 2010. Plant species and ecosystems with high conservation priority in Benin. In: van der Burgt X., van der Maesen L.J.G. \& Onana J.-M., eds. Systematics and conservation of African plants. Kew, UK: Royal Botanic Gardens, 429-444.

Ahmad S.S. et al., 2009. Prioritization of medicinal plants of Margala Hills National Park, Islamabad on the basis of available information. Pak. J. Bot., 41(5), 2105-2114.

Akoègninou A., van der Burg W.J. \& van der Maesen L.J.G., 2006. Flore analytique du Bénin. Wageningen, The Netherlands: Backhuys Publishers.

Arbonnier M., 2000. Arbres, arbustes et lianes des zones sèches d'Afrique de l'Ouest. Versailles, France : Quæ.

Barazani O., Perevolotsky A. \& Hadas R., 2008. A problem of the rich: prioritizing local plant genetic resources for $e x$ situ conservation in Israel. Biol. Conserv., 141(2), 596-600.

Barshila I., Devkota N.R. \& Bassila S.R., 2013. Perception of smallholder farmers on fodder tree utilization and management for livestock production in the mid-hills of Nepal. J. Anim. Prod. Adv., 3(10), 290-300.

Baumer M., 1997. L'agroforesterie pour les productions animales. Wageningen, Pays-Bas : CTA.

Bechir A.B., Mopate L.Y. \& Kabore-Zoungrana C.Y., 2009. Évaluation de la disponibilité saisonnière du fourrage ligneux en zone soudanienne du Tchad : cas du terroir de N'Guetté 1. Int. J. Biol. Chem. Sci., 3(1), 135-146.

Bechir A.B. \& Kabore-Zoungrana C., 2012. Fourrages ligneux des savanes du Tchad : structure démographique et exploitations pastorales. Cameroon J.Exp. Biol., 8(1), 35-46.

Brehm J.M. et al., 2010. New approaches for establishing conservation priorities for socio-economically important plant species. Biodiv. Conserv., 19(9), 2715-2740.

Brisso N., Houinato M., Adandédjan C. \& Sinsin B., 2007. Dry season woody fodder productivity in savannas. Ghanaian J. Anim. Sci., 2-3(1), 181-185.

Dagnelie P., 1998. Statistiques théoriques et appliquées. Bruxelles : De Boeck et Larcier.

Dassou H.G. et al., 2014. Diversité, usages vétérinaires et vulnérabilité des plantes médicinales au Nord-Bénin. Int. J. Biol. Chem. Sci., 8(1), 189-210.

de Souza S., 2008. Flore du Bénin: noms des plantes dans les langues nationales béninoises. Tome 3. $2^{\mathrm{e}}$ éd. Cotonou, Bénin : Imprimerie Tundé.

Egbe E.A., Tabot P.T. \& Fonge B.A., 2012. Ethnobotany and prioritization of some selected tree species in southwestern Cameroon. Ethnobot. Res. Appl., 10, 235-246.

FAO, 2009. La situation mondiale de l'alimentation et de l'agriculture : le point sur l'élevage. Rome : FAO.

Gaoue O.G. \& Ticktin T., 2007. Patterns of harvesting nontimber forest product from the multipurpose tree Khaya senegalensis in Benin: variation across climatic regions and its impacts on population structure. Biol. Conserv., 137, 424-436.

Geta T., Nigatu L. \& Animut G., 2014. Evaluation of potential yield and chemical composition of selected indigenous multi-purpose fodder trees in three districts of Wolayta Zone, Southern Ethiopia. World Appl. Sci. J., 31(3), 399-405.

Hahn-Hadjali K. \& Thiombiano A., 2000. Perception des espèces en voie de disparition en milieu gourmantché (Est du Burkina Faso). Ber. Sonderforschungsbereichs, 268(14), 285-297.

Hiernaux P., 2000. Implication of the "New Rangeland Paradigm" for natural resource management. In: Adriansen H., Reenber A. \& Nielsen I., eds. Proceedings of the 12 ${ }^{\text {th }}$ Danish Sahel Workshop, The Sahel: energy supply, economic pillars of rural Sahelian communities, need for revised development strategies, 3-5 January 2000. Copenhagen: SEREIN, 113-142.

Hiernaux P., Cisse M.I., Diara L. \& De Leeuw P.N., 1994. Fluctuations saisonnières de la feuillaison des arbres et des buissons sahéliens, conséquences pour la quantification des ressources fourragères. Rev. Élev. Méd. Vét. Pays Trop., 47, 117-125.

Houéhanou T.D. et al., 2008. Gestion pastorale et structure des terroirs agricoles dans la périphérie de la Djona (Nord-Est Bénin). Int. J. Biol. Chem. Sci., 2(4), 497-507.

Houinato M., 2001. Phytosociologie, écologie, production et capacité de charge des formations végétales pâturées dans la région des Monts Kouffé (Bénin). Thèse de doctorat: Université libre de Bruxelle (Belgique).

Hussain A. et al., 2013. Van Panchyats in Uttarakhand Himalaya-Lessons for conservation and sustainable 
utilization of forest resources. Int. J. Environ. Biol., 3(4), 208-217.

Idohou R. et al., 2013. National inventory and prioritization of crop wild relatives: case study for Benin. Genet. Resour. Crop Evol., 60, 1337-1352.

INSAE, 2013. Résultats provisoires de la quatrième phase du recensement général de la population et de l'habitation. Cotonou, Bénin : INSAE.

Joshi H.C. \& Samant S.S., 2004. Assessment of forest vegetation and prioritization of communities for conservation in a part of Nanda Devi Biosphere Reserve, West Himalaya, India. Part I. Int. J. Sustain. Dev. World Ecol., 11(3), 326-336.

Kabore-Zoungrana C.Y., 1995. Composition chimique et valeur nutritive des herbacées et ligneux des pâturages naturels soudaniens et des sous-produits du Burkina Faso. Thèse de doctorat d'État ès Sciences Naturelles : Université de Ouagadougou (Burkina Faso).

Kamuanga M.J. et al., 2008. Élevage et marché régional au Sahel et en Afrique de l'Ouest. Potentialités et défis. Paris : CSAO-OCDE/CE DEAO.

Maxted N. et al., 2006. Towards a definition of a crop wild relative. Biodivers. Conserv., 15(8), 2673-2685.

MDR (Ministère du Développement Rural), 1994. Définition d'une stratégie et d'un plan d'action pour le secteur de l'élevage. Volume 1 : rapport principal MDR. Cotonou, Bénin : MDR.

N'danikou S., Achigan-Dako E. \& Wong J.L.G., 2011. Eliciting local values of wild edible plants in Southern Benin to identify priority species for conservation. Econ. Bot., 65(4), 381-395.

Ndiaye M.,Dione M.E.\& Akpo L.E., 2010.Caractéristiques des ligneux dans les terroirs pastoraux de Ranérou (région de Matam, nord-Sénégal). J. Sci., 10(3), 12-27.

Onana J., 1995. Les ligneux fourragers du Nord-Cameroun. I. Inventaire et phénologie. Rev. Élev. Méd. Vét. Pays Trop., 48(2), 213-219.

Onana J. \& Devineau J.-L., 2002. Afzelia africana Smith ex Persoon dans le Nord-Cameroun. État actuel des peuplements et utilisation pastorale. Rev.Élev. Méd. Vét. Pays Trop., 55(1), 39-45.

Pant S. \& Samant S.S., 2007. Assessment of plant diversity and prioritization of communities for conservation in Mornaula Reserve Forest. Appl. Ecol. Environ. Res., 5(2), 123-138.

Petit S. \& Mallet B., 2000. L'émondage des arbres fourragers : détail d'une pratique. Bois For. Trop., 270, $35-45$.

Rawat S.Y. \& Vishvakarma C.R.S., 2011. Diversity, distribution and utilization of fodder species in subtemperate, temperate and cold desert region of the Himachal Pradesh, north-western, Himalaya. Int. J. Agric. Environ., 2, 23-32.

Robertson M.P. et al., 2003. A proposed prioritization system for the management of invasive alien plants in South Africa. South Afr. J. Sci., 99, 37-43.
Roothaert R.L. \& Franzel S., 2001. Farmers' preferences and use of local fodder trees and shrubs in Kenya. Agrofor. Syst., 52, 239-252.

Saha D. \& Sundriyal R.C., 2010. Priorization of non-timber forest produces for income generation: a participatory approach. J. Non-Timber For. Prod., 17(4), 387-394.

Sanon H.O., Kaboré-Zoungrana C. \& Ledin I., 2008. Nutritive value and voluntary feed intake by goats of three browse fodder species in the Sahelian zone of West Africa. Anim. Feed Sci. Technol., 144(1-2), 97-110.

Sanou B., 2014. Potentiel des ligneux fourragers du terroir de Sokouraba. Mémoire de Master en Productions et Industries animales: Institut du Développement Rural de l'Université Polytechnique Bobo-Dioulasso (Burkina Faso).

Sarr O. et al., 2013. Importance des ligneux fourragers dans un système agropastoral au Sénégal (Afrique de l'Ouest). Rev. Méd. Vét., 164(1), 2-8.

Shenkute B. et al., 2012. Identification and nutritive value of potential fodder trees and shrubs in the mid rift valley of Ethiopia. J. Anim. Plant Sci., 22(4), 1126-1132

Silue N. et al., 2014. Identification des espèces ligneuses utilisées dans l'alimentation des bovins dans la région du Poro (nord de la Côte d'Ivoire). Agron. Afr., 26(3), 217-229.

Singh A. \& Samant S.S., 2010. Conservation prioritization of habitats and forest communities in the Lahaul Valley of proposed cold desert biosphere reserve, north western Himalaya, India. Appl. Ecol. Environ. Res., 8(2), 101117.

Sinsin B. \& Kampman D., 2010. Atlas de la biodiversité de l'Afrique de l'Ouest. Tome 1 : Bénin. Cotonou, Bénin ; Frankfurt/Main, Allemagne : BIOTA.

Soulama S. et al.,2013. Teneurs en coumarines de 15 ligneux fourragers du Burkina Faso. Int. J. Biol.Chem.Sci., 7(6), 2283-2291.

Teka O., Vogt J. \& Sinsin B., 2007. Impacts de l'élevage sur les ligneux fourragers et contribution à la gestion intégrée de Khaya senegalensis et Afzelia africana, deux espèces menacées d'extinction dans la région des Monts-Kouffé au Bénin. Bull. Rech. Agron. Bénin, 55, 25-35.

Tukur R., Adamu G.K.N. \& Abdulrahid I., 2013. Indigenous trees inventory and their multipurpose uses in DutsinMa area Katsina State. Eur. Sci. J., 9(11), 1857-1881.

Vodouhê F.G., Coulibaly O., Greene C. \& Sinsin B., 2009. Estimating the local value of non-timber forest products to Pendjari Biosphere Reserve Dwellers in Benin. Econ. Bot., 63(4), 397-412.

White F., 1983. The vegetation map of Africa south of the Sahara. $2^{\text {nd }}$ ed. Paris : UNESCO.

Zuur A.F., Ieno E.N. \& Elphick C.S., 2010. A protocol for data exploration to avoid common statistical problems. Methods Ecol. Evol., 1, 3-14.

(57 réf.) 